\title{
Numerical Simulation of Circular Cylinders in Free-Fall
}

\author{
Pedro Romero-Gomez, Marshall C. Richmond* \\ Hydrology Group, Pacific Northwest National Laboratory, Richland, WA, USA 99352
}

\begin{abstract}
In this work, we combined the use of (i) overset meshes, (ii) a 6 degree-of-freedom (6DOF) motion solver, and (iii) an eddy-resolving flow simulation approach to resolve the drag and secondary movement of large-sized cylinders settling in a quiescent fluid at moderate terminal Reynolds numbers $(1,500<\operatorname{Re}<28,000)$. These three strategies were implemented in a series of computational fluid dynamics (CFD) solutions to describe the fluid-structure interactions and the resulting effects on the cylinder motion. Using the drag coefficient, oscillation period, and maximum angular displacement as baselines, the findings show good agreement between the present CFD results and corresponding data of published laboratory experiments. We discussed the computational expense incurred in using the present modeling approach. We also conducted a preceding simulation of flow past a fixed cylinder at $\operatorname{Re}=3,900$, which tested the influence of the turbulence approach (time-averaging vs eddy-resolving) and the meshing strategy (continuous vs. overset) on the numerical results. The outputs indicated a strong effect of the former and an insignificant influence of the latter. The long-term motivation for the present study is the need to understand the motion of an autonomous sensor of cylindrical shape used to measure responses to the hydraulic conditions occurring in operating hydropower turbines.
\end{abstract}

Keywords:

Overset grids, cylinder, drag, secondary motion, 6-DOF, CFD

\section{Introduction}

The motion of particles in fluid flow is important in a wide variety of phenomena involving particle movement in nature, machinery, chemical processing, and civil infrastructure. Studies have examined a wide variety of particle shapes (spheres, ellipsoids, and cylinders) in various flow conditions (quiescent fluid, laminar, or turbulent flows). From the perspective of applied technology, the interest in examining fluid dynamic forces and the resulting motion of falling cylinders has come from various fields. Seminal work can be found in McNown and Malaika (1950). A brief list of applications includes chemical process devices such as classifiers, dust collectors, and conveyors (Christiansen and Barker, 1965); environmental studies of erodibility, movement, suspension, and deposition of earth material (Dietrich, 1982); biomass particles transforming into fuel and chemicals (Ren et al., 2011); and carbon sequestration in deep waters

\footnotetext{
* Corresponding author

Email address: marshall.richmond@pnnl.gov (Marshall C. Richmond) 
using field hydrate particles (Chow and Adams, 2011). The present work is motivated by the long-term goal of simulating the motion of an autonomous sensor device of cylindrical shape used in hydropower turbine investigations (Carlson et al., 2003, Deng et al., 2007) to record the device response to hydraulic conditions. This work reports the first stage towards our ultimate goal, and consisted of a numerical study of the motion of simulated cylinders in quiescent fluid conditions, a canonical test case that minimized the complexities associated with highly turbulent flows in hydro-turbines. For that reason, the literature, simulations and discussions herein focused on cylinders of large length to diameter ratio (aspect), rather than on disks or oblate ellipsoidal particles, which can exhibit fundamentally different patterns of motion.

Laboratory studies have long demonstrated that the parameters characterizing the free-falling cylinder motion depended on the motion regime itself. For instance, early authors carried over the knowledge basis from fluid flows past fixed cylinders, and for the most part characterized the particle motion regimes and surrounding flow conditions as a function of Reynolds number (Re, defined in terms of the cylinder diameter and terminal velocity). Settling cylinders were therefore observed to maintain their release orientation and to lack oscillatory motion in the Stokes regime $(\operatorname{Re}<0.01)$ (Jayaweera and Mason, 1965), but showed preferential orientation exposing the largest projected area to the direction of the motion (e.g., cylinder axis in horizontal position for ratios length-to-diameter $>1)$ in the so-called transition range $(0.01<\mathrm{Re}<100)$ (Marchildon et al., 1964). Although eddies appeared in the wake flow at $\operatorname{Re}>1$ and a Kármán vortex shedding developed at $\operatorname{Re}>50$, no oscillatory motion of the body was observed. Values of $\operatorname{Re}$ $>100$ triggered a secondary motion characterized by the cylinder's main axis oscillating in the vertical plane, and the centroid's trajectory oscillating around the mean falling path (Clift et al., 2005). The onset of secondary motion was also reported at $\operatorname{Re}>400$ in an extensive laboratory work with cylindrical and irregular-shaped particles (Stringham et al., 1969). Within moderate Reynolds number $(100<\operatorname{Re}<2,400)$, the particle oscillations were very regular and allowed for determining the oscillation time and maximum angular deflection as a function of the length-to-diameter and particle-to-fluid density ratios (Marchildon et al., 1964, Isaacs and Thodos, 1967, Chow and Adams, 2011), i.e. Reynolds number alone cannot characterize secondary particle motion. The motion regularity was found to be valid for an extended range $(200<\mathrm{Re}$ $<$ 6,000) (Chow and Adams, 2011). The present study focused on this moderate Re range and on body length to diameter ratios greater than one. For larger Reynolds numbers, the oscillations became three-dimensional, and this complexity precluded the development of empirical relationships for secondary motion. Drag coefficients showed no correlation with Reynolds numbers as high as 300,000 (Christiansen and Barker, 1965, Isaacs and Thodos, 1967), which suggested the need for other dimensionless parameters such as the aspect ratio and specific gravity of the cylinders to characterize the terminal velocity (Marchildon et al., 1964, Chow and Adams, 2011).

Further studies have sought to gain understanding of particle motion in more complex flow configurations. For instance, falling cylinders subject to an upward gas flow have also been examined (Ren et al., 2011), and the influence of both the initial drop conditions and center-of-mass location has been tested (Chu et al., 2005). Other works have analyzed the secondary motion from an analytical perspective, suggesting that the oscillations depend on the non-coincidence between the center of pressure and center of gravity of the particle (Mandø and Rosendahl, 2010). Computational fluid dynamics (CFD) runs simulated the flow fields of horizontal and inclined particles of cylindrical shape, but orientations were fixed (Hashino et al., 2014).

The present study of the motion of a cylinder in free-fall will support subsequent studies to determine how an autonomous sensor device moves through and responds to hydro-turbine flows (Carlson et al., 2003, Deng et al., 2007). Because these responses can be related to injuries of 
fish passing through hydropower turbines in operation, the environmental performance of new and replacement turbines can be both evaluated and improved (Richmond et al., 2009). The present work represents a canonical test case that allows us to perform an initial validation of a virtual version of the autonomous sensor where the complicating factors present in hydropower turbine systems are not present, and one for which experimental data are readily available. We envision a future modeling approach that consists of using an overset moving mesh to represent the autonomous sensor traveling through the rapidly changing hydropower turbine flows, thereby improving the state-of-art design tools described by Richmond et al. (2014). The present work constitutes the first step for determining the accuracy of simulating the drag and secondary motion of an equivalent cylinder in a quiescent fluid environment.

In this work we use a moving overset mesh scheme containing the cylinder as a means to simulate the dynamic flow fields arising around the settling cylinder. We present a general description of the overset mesh approach and its implementation in the commercial CFD code used to conduct this study. Before simulating cylinders in free-fall, we tested the overset mesh capability in a fixed cylinder at a condition relevant to the cases in motion $(\operatorname{Re}=3,900)$. These initial numerical tests helped to quantify the effect of using an overset, discontinuous mesh, instead of a single continuous grid as conventionally used. The eddy-resolving turbulence model is briefly described, as well as its performance in comparison to conventional two-equation turbulence modeling. Then, the simulations of freely settling cylinders are described, and results for drag coefficients and secondary motion are presented. We also discuss the computational effort required to accomplish the task, which still remains an issue when considering using the overset mesh approach for this and other similar engineering applications for moving bodies. We particularly emphasize the relevance of the selected cases to the target application, i.e., to understand the hydrodynamics of the moving autonomous sensor.

\section{Methods}

\subsection{The overset mesh scheme}

The use of overset meshes-also known as chimera or overlapping grids-in CFD simulations has gradually evolved since its introduction to solve simple benchmarking flow cases (Dougherty, 1985, Hinatsu and Ferziger, 1991). Conceptually, this approach consists of combining a system of grids to accommodate irregular domains or grids with different characteristics (rectangular and cylindrical grids, for instance) (Ferziger and Perić, 2002). This feature is particularly useful for simulating domains that cannot be represented by a single, continuous, fixed mesh because of geometric irregularities or dynamic changes in response to the surrounding flow conditions. One advantage that has made this strategy increasingly attractive for transient CFD simulations is that the composed mesh does not have to be regenerated at each time step, thereby reducing the computational effort in comparison to other techniques such as mesh morphing. However, some computational effort must be spent on calculating the flow quantities at the interface where interacting grids communicate with each other (CD-adapco, 2014).

In the present application, we used the commercial code STAR-CCM+v9 to generate cylinders fully contained within a larger domain of hexahedral cells (CD-adapco, 2014). Figure 1 depicts a schematic of the mesh-overlapping strategy. The cylindrical body was confined within a fluid volume (bounded by the black rectangle), which was referred to as the overset mesh. This volume was then discretized, and inserted into the main computational domain representing the flow environment (the background mesh, blue region). The computation of the fluid 
flow proceeded simultaneously in both implicitly coupled meshes-much as with a continuous mesh-with the exception that the solver then identified a number of outer layers of overset cells (overlapping cells, in green) on which the flow solution was not computed from conservation equations but instead was linearly interpolated from both the overset and background meshes. Because this strategy creates discontinuity between the two regions, the software offers a number of interpolation models that have been proven to retain the flow physics to a large extent, namely, linear, distance-weighted, and least-square schemes.

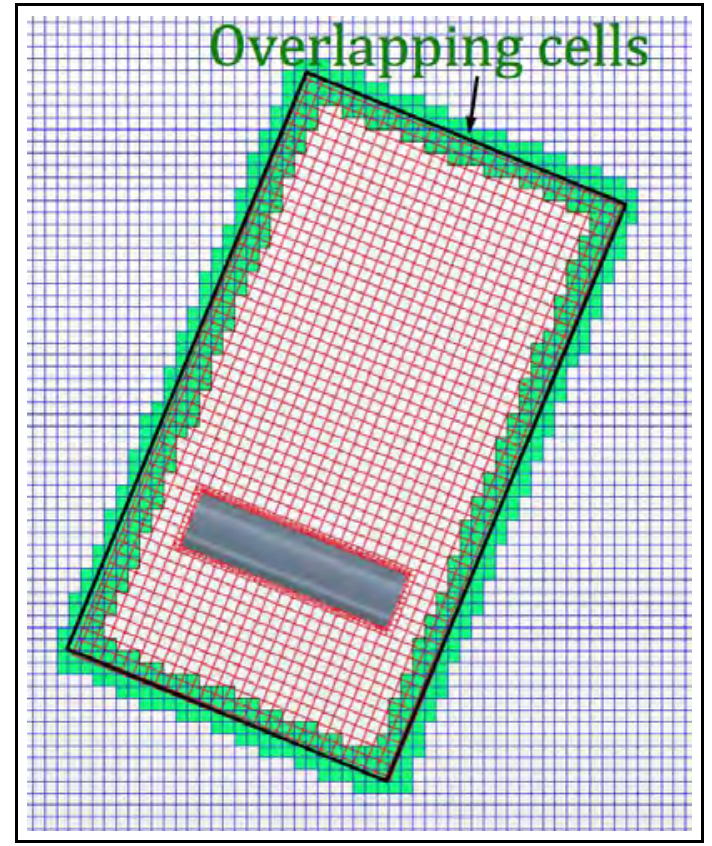

Figure 1: The overset mesh scheme. The blue mesh correspondes to the background, the red mesh to the overset, and the green region represent the overlapping cells

To assemble the two regions at the green cells in Fig. 1, the algorithm follows two steps: (i) the hole-cutting step to eliminate cells that do not take part in the flow field calculation, and (ii) the donor cell search to determine which cells contribute to the variable calculation at an "acceptor" cell. In the schematic in Fig. 2, the background mesh is in blue, and the overset is in red. The central aspect of using an overset mesh consists of computing flow quantities at the last active overset cell $(\mathrm{C}$, red cell) based on the usual algebraic representation of the conservation equations, but interpolating them at the acceptor overset cells (A, hollow circle). The donor search locates those background cells bounding the acceptor overset cell (i.e., the search identifies $\mathrm{N}_{4}, \mathrm{~N}_{5}$, and $\mathrm{N}_{6}$, the vertices in the dashed-line triangle). The actual set of donor cells depends on the interpolation technique, which was selected to be a linear in this study. The flow quantity at the cell center $\mathrm{A}\left(\phi_{A}\right)$ is then calculated as $\phi_{A}=\sum \alpha_{i} \phi_{N_{i}}$, where $\alpha_{i}$ are the interpolation weighting factors, and $\phi_{N_{i}}$ are the flow quantity values at the located donor cells. The flow quantities at $\mathrm{C}$ are solved as usual, and involve the three neighboring cells from the overset $\left(\mathrm{N}_{1}, \mathrm{~N}_{2}\right.$, and $\left.\mathrm{N}_{3}\right)$ and the background meshes $\left(\mathrm{N}_{4}, \mathrm{~N}_{5}\right.$, and $\left.\mathrm{N}_{6}\right)$. The fluxes through the cell face (between A and C) are computed in a manner similar as for two active cells. On 
the opposite end of the overlapping cell layer, the calculation procedure is the same, but now the acceptor cells belong to the background mesh and the flow quantities are interpolated from donor cells pertaining to the overset mesh.

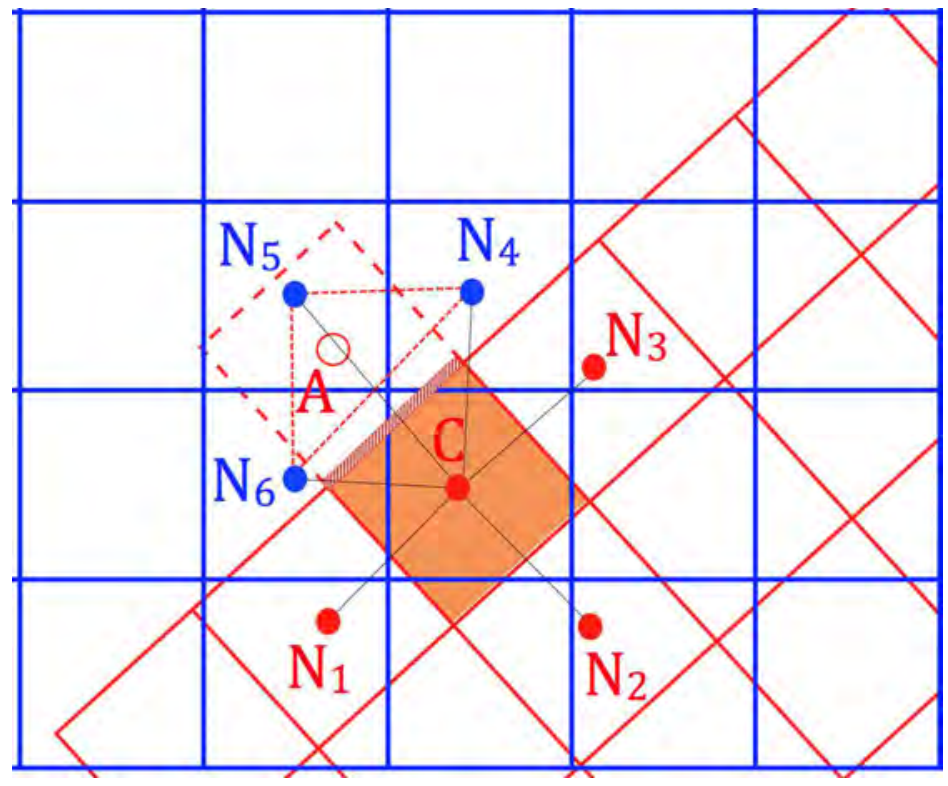

Figure 2: The interpolation scheme.

\subsection{Eddy-resolving turbulence modeling}

In this study, the flows arising around the cylinders in free-fall of this study are turbulent, i.e. they vary considerably in both position and time. Conventionally, the treatment of turbulence in CFD simulations entails the use of a "turbulence closure" formulation, which dictates whether most velocity fluctuations are represented by a surrogate quantity such as the turbulent kinetic energy (turbulence-averaging techniques) or are explicitly resolved (eddy-resolving techniques). We used the two approaches by solving the flows past a fixed cylinder with both the unsteady Reynolds-averaged Navier-Stokes equations (URANS) and detached eddy simulation (DES). The URANS technique derives from time-averaging the conservation equations, and from calculating the turbulence effects on the mean flow through the so-called Reynolds stresses. A DES models the turbulent quantities in the "attached" portion of the flows (similar to URANS near the walls) and resolves the large eddies in the core flow ("detached" flow). The technical literature related to the development and application of DES methods is abundant and can provide more insight into both turbulence modeling strategies (Spalart, 2009). For the simulation of cylinders in free-fall, we exclusively used only the DES turbulence model.

We used both the URANS and DES version of the $k-\omega$ SST (shear stress transport) turbulence model, which are available in the CFD software. The turbulence model was originally developed by Menter (1994), and - similar to the other two-equation models-assumes that the Reynolds stresses emerging from turbulence fluctuations are proportional to the mean velocity gradients. This turbulence model is implemented in the software in the form of two transport 
equations that are solved simultaneously with the mass and momentum conservation equations to calculate the velocity field. In the conservation equation in Eq. 1, $\phi$ takes the form of two turbulent quantities, namely, the turbulent kinetic energy $(k)$ and its specific rate of dissipation $(\omega)$. Because the conservation principle is applied for these quantities within a finite volume $(\mathrm{dV})$ bounded by surfaces $(\mathrm{d} \bar{a})$, their transient changes (first term, LHS) and convective fluxes (second term, LHS) are a product of diffusion fluxes (first term, RHS) and internal sources (second term, RHS). Notice that in addition to the dynamic viscosity $(\mu)$, the turbulent viscosity $\left(\mu_{t}\right)$ contributes to the diffusion processes. In turbulent flows, this turbulent viscosity is many orders of magnitude larger than the fluid property $(\mu)$, and is itself a function of $k$ and $\omega$. The parameter $\mathrm{P}_{\phi}$ groups the dissipation, dispersion and production of the turbulent quantities, which represent the core distinction between the URANS and DES versions. In the Eq. 1, $\rho_{w}$ is the fluid density, $\sigma_{k}$ and $\sigma_{\omega}$ are the inverse turbulent Schmidt numbers, and $\bar{u}$ and $\bar{u}_{g}$ are the fluid and grid velocities, respectively. Menter and Kuntz (2004) described the development of the DES version that sought to enhance prediction accuracy of simulations of unsteady separated flows behind moving vehicles. The full description of the equations can be found in the software manual (CD-adapco, 2014), and the time-scale coefficient $\left(\mathrm{C}_{d e s T}\right)$ for the blended upwind scheme was set to a value of 0.1 as in the original formulation (Travin et al., 2002).

$$
\frac{d}{d t} \int_{V} \rho_{w} \phi \mathrm{d} V+\int_{A} \rho_{w} \phi\left(\bar{u}-\bar{u}_{g}\right) \cdot \mathrm{d} \bar{a}=\int_{A}\left(\mu+\sigma_{\phi} \mu_{t}\right) \nabla \phi \cdot \mathrm{d} \bar{a}+\int_{V} P_{\phi} \mathrm{d} V
$$

\subsection{6-DOF motion solver}

The overset mesh containing the cylinder was set to free motion in all linear and angular directions. Therefore, linear motion of the center of mass of the cylinder is governed by Eq. 2 solved in the global inertial frame of reference, whereas the rotational motion is formulated in the relative frame of the body with origin in its prescribed center of mass (Eq. 3).

$$
\begin{gathered}
m \frac{d \bar{v}}{d t}=\bar{f} \\
\overline{\bar{M}} \frac{d \omega}{d t}+\bar{\omega} \times \overline{\bar{M}} \bar{\omega}=\bar{n}
\end{gathered}
$$

At each time step, the solver computed the vector $\bar{f}$ as the resultant from the pressure, shear and gravity forces acting on the cylinder surface, and $\bar{n}$ as the resultant from the pressure and shear moments acting on the body. The solver then computed the linear $(\bar{v})$ and angular $(\bar{\omega})$ velocities, to finally bring the body location and angular position to the new state. In the process, the body affected the surrounding flow conditions, which made the motion solver strongly coupled with the flow calculation. The tensor of moment of inertia $(\overline{\bar{M}})$ and body mass (m) were prescribed in the simulation.

Initial angular and linear velocities were set equal to zero, and the moment of inertia around the center of mass was stipulated to be the same as for a solid cylinder. A maximum of four iterations were set to advance the 6-DOF body to the new state. Along the trajectory, we monitored the cylinder's centroid location, the angular position, and the linear and angular velocities. The time series of these monitored quantities were used to determine the terminal velocity and oscillation periods for each simulated case, as well as the maximum inclination angle of the cylinder during its descend. More information about the formulation of 6-DOF motion solver can be found in the software manual (CD-adapco, 2014). 


\section{Simulations}

\subsection{Flow past a fixed cylinder at $\operatorname{Re}=3,900$}

We simulated the fluid flow past a fixed cylinder as a precursor to first determine the most adequate settings to be used later in the simulation of cylinders in free-fall without the complicating factors of free $6 \mathrm{DOF}$ motion. We particularly tested three settings relevant to the numerical solution of settling cylinder motion: the turbulence approach (URANS vs. DES), the meshing strategy (continuous vs. overset grids), and the use of Cartesian vs. boundary-fitted grids. Flow past a stationary cylinder is one test case with a long history in the field of fluid dynamics. The main reasons for its popularity are both the simplicity of its geometry and the different behaviors flow exhibits depending on the Reynolds number. The value of $\mathrm{Re}=3,900$ is a widely analyzed case in the so-called subcritical flow regime, and is relevant for the expected range of Reynolds numbers that the autonomous sensor encounters during its movement through hydro-turbine intake flows. Although the literature on the flow past a cylinder at $\operatorname{Re}=3,900$ is abundant, in this work we conducted our study mostly based on three references that thoroughly tested numerical formulations to describe the flow features (Kravchenko and Moin, 2000, Dong et al., 2006, Luo et al., 2014). Large eddy simulations with the Galerkin B-spline method were run on Otype meshes of various densities and span-wise lengths (Kravchenko and Moin, 2000). DES and Partially Averaged Navier-Stokes (PANS) solutions were also implemented on O-type meshes and compared against published laboratory data (Luo et al., 2014). In addition, direct numerical simulations (DNS) with a Fourier spectral expansion were implemented in Cartesian domains (box-type), and compared against new PIV data showing detailed features of the structures in the near wake flow (Dong et al., 2006).

In this study, the cylinder of diameter $D$ was set up in a box-type computational domain spanning - $15 \mathrm{D}$ to $25 \mathrm{D}$ in the direction of the flow, $-9 \mathrm{D}$ to $9 \mathrm{D}$ in the vertical direction, and $-\pi / 2$ to $\pi / 2$ diameters in the direction of the main axis of the cylinder. The latter was found to be sufficient to resolve the three-dimensional structures arising from the cylinder surface (Dong et al., 2006). Based on the friction velocity at the top tip of the cylinder $\left(u^{*}\right)$, the dimensions of the first layer of cells off the cylinder surface were $0.4,2.4$ and 7.0 wall-units in the radial, azimuthal, and spanwise directions, respectively. The mesh was gradually coarsened away from the cylinder, with core cells of approximately 48 wall units. The mesh size was $1.96 \mathrm{M}$ cells, and the cell distribution is depicted in Fig. 3. The CFD software used a finite-volume method for transient solutions with both the URANS and DES versions of the $k-\omega$ SST turbulence model. The upstream inflow condition was set as a constant velocity $\left(U_{0}\right)$ with very low turbulence intensity $(1 \%)$. The top and bottom boundaries were set as symmetry planes, whereas the lateral walls were set as periodic boundaries. The "warm-up" simulation period was approximately 320 convective times $\left(D / U_{0}\right)$, and statistics were collected thereafter for a period of 400 convective times. In DES (case B in Table 1), the temporal discretization was set as second-order, and the transient solution was advanced at intervals of $\Delta t U_{0} / D=0.034$. The segregated flow solver was combined with a hybrid second-order upwind/central differencing convection scheme. For the URANS simulation (case A in Table 1), the domain extents, mesh and boundary conditions remain the same as for the DES, but the time step was made 4 times longer than in DES $\left(\Delta t U_{0} / D=0.1354\right)$, and the convection scheme was set as second-order upwind. Because cases A and B were essentially the same except for the turbulence modeling approach, the output of this test was the selection of the most accurate turbulence treatment to be used in the subsequent settling cylinder cases. 
In the overset mesh solution (case C), a domain containing the cylinder was $-3 \mathrm{D}$ to $8 \mathrm{D}$ in the stream-wise direction, $-3 \mathrm{D}$ to $3 \mathrm{D}$ in the vertical direction, and the same span-wise length as the background (see Fig. 3). All other features remained the same as for case B, thereby allowing us to test the effects of an overset mesh strategy on the numerical solution. The two lateral overset boundaries (that were periodic) coincided with the two lateral background boundaries (also periodic), and for that reason, the "overset boundary" topology was set to distinguish between those overset boundaries that were "closed" (e.g., in the direction from inflow to outflow, and from bottom to top) from those that coincided with the background boundaries (lateral periodic boundaries).

Finally, we also ran a DES simulation on a conventional O-type mesh (case D) generated with pro-STAR (a CD-adapco product). The extents, densities, and other features of the mesh followed those of a previous study (Luo et al., 2014). The outer boundary condition was set as a constant velocity with fixed direction, and a low turbulence intensity $(1 \%)$. The flow solver settings were the same as in cases B and C. Because the settling cylinder simulations were conducted in a Cartesian domain but flow past cylinders has been conventionally solved in O-type meshes, comparisons between cases $\mathrm{B}$ and $\mathrm{D}$ allowed us to establish the differences in solutions obtained using meshes with different topologies.

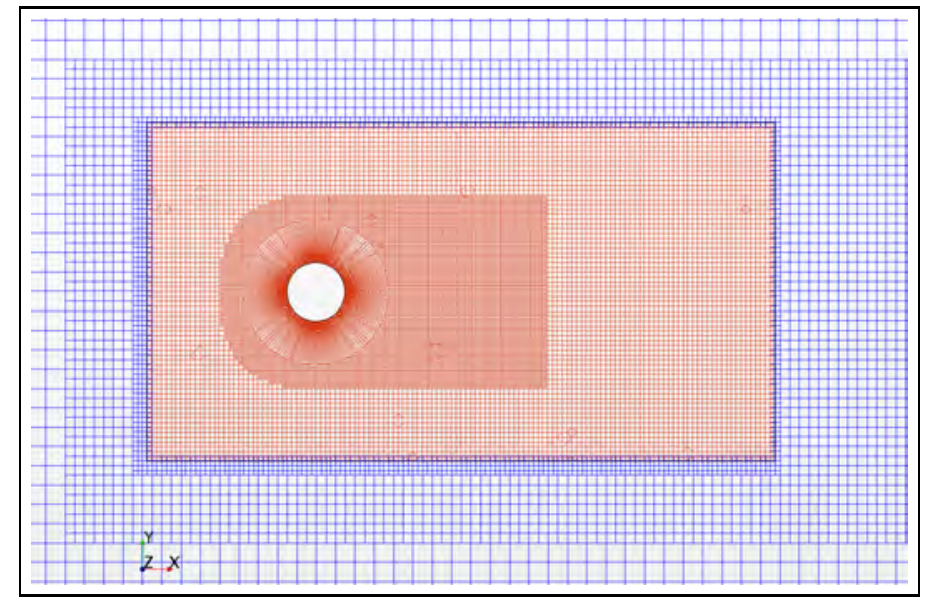

Figure 3: Cross-sectional view of the background (blue) and overset (red) meshes to simulate the flow past a fixed cylinder at $\operatorname{Re}=3,900$.

Table 1 presents a summary of the flow parameters from the presently implemented solutions, as well as previous numerical studies. The URANS formulation overestimated both the drag coefficient $C_{D}$ and the mean base pressure coefficient $\left(-C_{b p}\right)$, but yielded an acceptable agreement in shedding frequency $(S t)$, especially with respect to the O-type mesh solution (case D). The URANS simulation largely suppressed the three-dimensionality of the flow, thereby influencing the pressure distribution on the cylinder surface, which ultimately had an impact on pressure coefficients. In addition, the RANS equations used empirical formulations for the Reynolds stresses that may not model the wide range of time and length scales present in the flow. These trends and arguments have been previously discussed in an overview of URANS and DES performance in flow past cylinders (Spalart, 2009), as well as in modeling studies specifically at $\operatorname{Re}=3,900$ (Young and Ooi, 2007, Shim et al., 2009). More important, the use of an overset mesh (case 
Table 1: Summary of flow parameters from previous and the present work.

\begin{tabular}{lcccc}
\hline Previous studies & $C_{D}$ & $-C_{b p}$ & $\mathrm{St}$ & $\theta_{\text {sep }}{ }^{\circ}$ \\
\hline Experimental (Norberg, 1987) & $0.98 \pm 0.05$ & $0.90 \pm 0.05$ & $0.215 \pm 0.005$ & $85.0 \pm 2.0$ \\
LES, O-type(Kravchenko and Moin, 2000) & 1.04 & 0.94 & 0.210 & 88.0 \\
DNS, box-type (Dong et al., 2006) & - & 0.96 & 0.210 & - \\
DES, O-type (Luo et al., 2014) & 1.10 & 1.01 & 0.203 & - \\
\hline Present solutions & & & & \\
\hline A, URANS, Continuous & 1.26 & 1.35 & 0.207 & 92.3 \\
B, DES, Continuous & 1.06 & 1.00 & 0.218 & 87.7 \\
C, DES, Overset & 1.07 & 1.01 & 0.211 & 86.8 \\
D, DES, O-type, Continuous & 1.02 & 0.93 & 0.207 & 87.3 \\
\hline
\end{tabular}

C) did not result in considerable loss of accuracy with respect to the continuous mesh (case B). The O-type mesh (case D) produced the lowest drag and mean base pressure coefficients that compare well with more advanced turbulence treatments such as LES and DNS.

The top contour plots in Fig. 4 present the normalized vorticity distributions from a previous study (Dong et al., 2006) and from cases B, C, and D. The contour plots captured the extents of the shear layer in the near wake as predicted or measured from all of the indicated approaches. Qualitatively, the three present numerical solutions acceptably reproduced the extents and general features of the shear layers, and solution D was the closest to the experimental PIV-based result. Both cases B and C eliminated the thick tip of the shear layer region present in the DNS solution. The bottom contour plots show the normalized Reynolds stress distribution exhibiting four "enclosing regions" in the PIV, DNS, and case C solutions (two small upstream nearly circular regions, and two multilayer regions behind them), but only the two major "enclosing regions" were present in case B and D solutions. Furthermore, the maximum Reynolds stresses (the innermost contours of the large "enclosing regions") were found further downstream in the experimental PIV results than in all of the modeling approaches.

The distribution of pressure coefficients on the cylinder surface (Fig. 5a) shows good performance for solution D (O-type mesh), an acceptable agreement from the two box-type solutions with DES (cases B and C), and the poor accuracy of solution A (URANS). The tendency to deviate from the experimental results was larger for URANS, which also yielded the most delayed separation point from all of the solutions (largest $\theta_{\text {sep }}$ in Table 1), and smaller for the three DES solutions with earlier separation angles. Other factors such as the cell size distribution on the cylinder surface and the growth rate in the radial direction affect the correlation of numerical results to experimental data. Figure $5 \mathrm{~b}$ shows the comparisons of normalized stream-wise velocity distributions at three transects in the near wake, whereas further transects are shown in Fig. 5c. The vertical scale of the former ranges from 0.0 to 1.0, whereas that of the latter ranges from 0.7 to 1.0 . The cylinder center's location corresponded to $x / D=0.0$. The velocity profile recovered faster in the URANS than in any DES solution because averaging the fluctuations tended to mix both the disturbed near wake flow and the ambient stream flow at a faster rate. DES runs, on the other hand, allowed the turbulent structures originating from the cylinder surface to be transported and propagated further downstream, giving rise to delayed mixing between the near-wake and stream flows. A slightly better agreement was observed in the box-type solutions (cases B and C) than in the O-type mesh (case D). This was likely related to the fact that although the mesh density of the former was coarsened by blocks, it maintained the cell density constant within each block, as opposed to the O-type mesh in which a growth factor was applied to gradually increase 


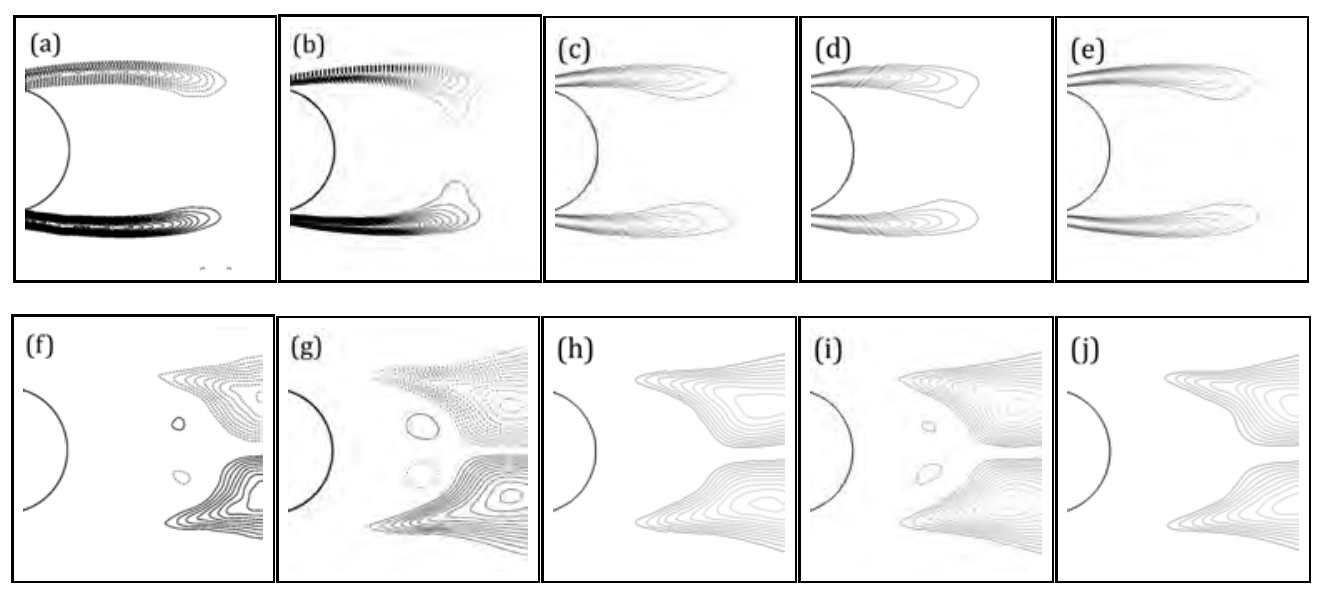

Figure 4: Contours of normalized span-wise vorticity (top, $\left|\bar{\omega}_{z} D / U_{0}\right|_{\min }=2.58$ and $\left|\Delta \bar{\omega}_{z} D / U_{0}\right|=0.64$ ) and normalized Reynolds stress (bottom, $\left|\left\langle u^{\prime} v^{\prime}\right\rangle / U_{0}^{2}\right|_{\min }=0.03$ and $\left|\Delta\left\langle u^{\prime} v^{\prime}\right\rangle / U_{0}^{2}\right|=0.01$ ): (a and f) PIV (Dong et al., 2006), (b and g) DNS (Dong et al., 2006), (c and h) DES, continuous mesh (case B), (d and i) DES, continuous mesh (case C), and (e and j) DES, O-type mesh (case D). In the present study, the flow fields were averaged over a period of 400 convective times
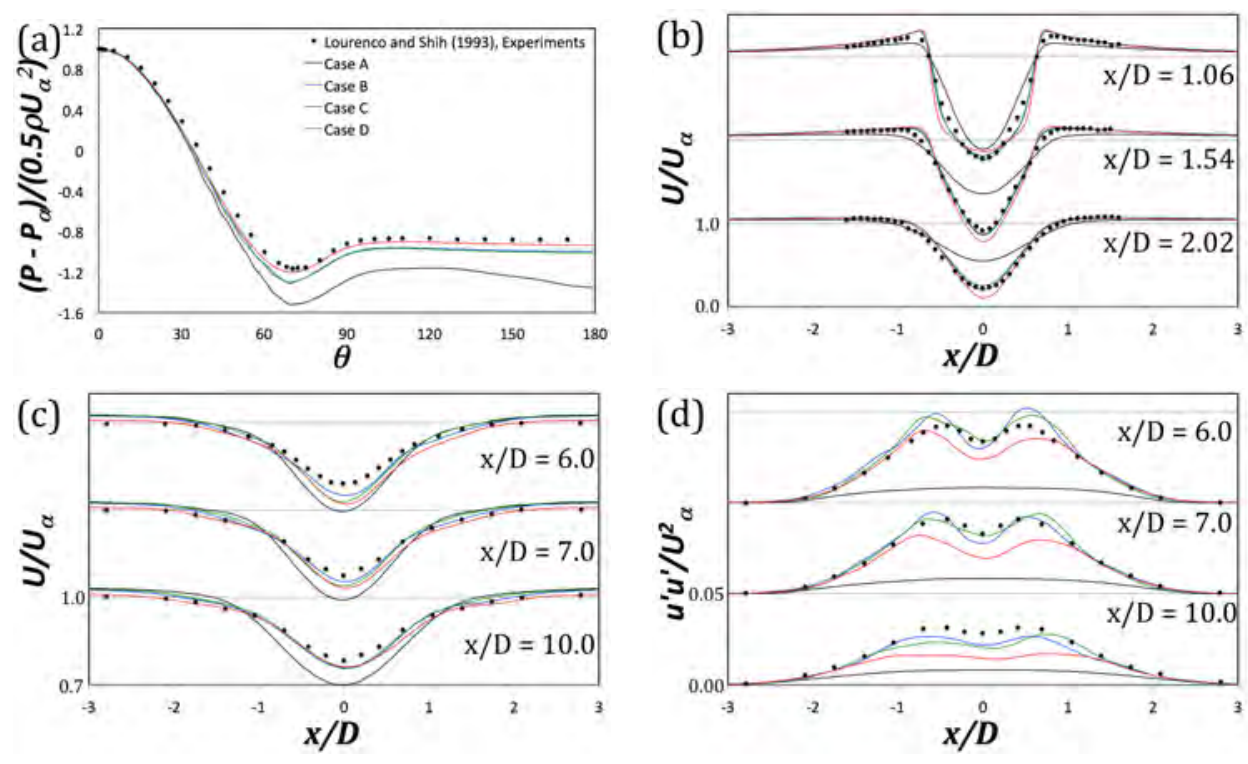

Figure 5: (a) Azimuthal distributions of pressure coefficient $\left(C_{P}\right)$ along the cylinder surface from the present data (all cases in table 1) and published laboratory data, (b) Profiles of normalized streamwise velocity at three locations at the near wake, (c) Profiles of normalized streamwise velocity at three locations further from the cylinder, and (d) Profiles of Reynolds stresses $\left(R e_{U U}\right)$ at three locations further from the cylinder.

the cell size at each subsequent layer in the radial direction. This fact limited the ability of the O-type solution to propagate the fluctuations as effectively as the box-type solutions, a situation that is also reflected in the better agreement of solutions B and C in terms of the Reynolds stress 
at the three far transects (Fig. 5d).

In summary, the stationary cylinder tests confirmed that DES was necessary to describe the flows surrounding settling cylinders, because URANS largely failed to accurately reproduce a number of flow parameters other than the shedding frequency (Strouhal number). In addition, the comparisons between solutions $\mathrm{B}$ and $\mathrm{C}$ indicated a limited effect of the discontinuity associated with overset meshes. Lastly, comparisons between solutions B and D suggest that boundaryfitted meshing strategies do not substantially increase numerical accuracy with respect to boxtype grids. This aspect is beneficial for simulating settling cylinders in very dynamic motions that modify the mesh configurations at each time step.

\subsection{Cylindrical particles in free-fall: drag coefficient}

By resolving cylinder motion with overset meshes, we targeted the initial development of capabilities to represent the movement of an autonomous sensor through hydro-turbines. When deployed in the field, this sensor package encounters various conditions ranging from mild flow acceleration shortly after release at the converging turbine intake, to highly dynamic vortex ropes in the draft tube. Based on the slip velocity, this wide variety of conditions results in a large range of Reynolds numbers to which the unit is subjected. Low Reynolds numbers $(\operatorname{Re}<1,500)$ could be present but they are not prevalent, whereas high Reynolds numbers $(\operatorname{Re}>30,000)$ may place during passage through the runner blade and the draft tube. Therefore, we chose to resolve the settling cylinder motion at the moderate $\operatorname{Re}$ values $(1,500<\operatorname{Re}<30,000)$ that are likely to be representative of conditions occurring as the sensor package travels from the point of release through the turbine.

A few studies have reported laboratory data of large cylinders in this moderate Re range. Specifically, the experimental Re value ranges were: 70 - 2,400, (Marchildon et al., 1964); 200 60,000 (Isaacs and Thodos, 1967); 1,000 - 300,000 (Christiansen and Barker, 1965); and $200-$ 6,000 (Chow and Adams, 2011). Most experimental materials were negatively buoyant in water and included (in increasing specific gravity) lucite (low density), nylon, resin, rubber, teflon, aluminum, polycarbonate, steel, brass, copper, lead, and gold. Only one positively buoyant material (wood) was reported (Marchildon et al., 1964). The cylinders were released in tanks containing water, and the terminal velocities were determined either with the inspection of images captured with cameras at set vertical stations (Marchildon et al., 1964, Isaacs and Thodos, 1967, Chow and Adams, 2011) or with the use of a photoelectric timer (Christiansen and Barker, 1965). The laboratory results were described and presented as a function of the cylinder aspect ratio $(E=L / D)$ and the density ratio $\left(S=\rho / \rho_{w}\right)$. We compared and judged the quality of the present numerical results based on drag coefficients calculated as in Eq. 4 , where $C_{d}$ is the drag coefficient; $\rho$ and $\rho_{w}$ are the cylinder and water densities, respectively; $u_{s}$ is the terminal velocity; and $g$ is the gravity acceleration. The laboratory measurement of $C_{d}$ was reported in terms of the Reynolds number (Marchildon et al., 1964, Isaacs and Thodos, 1967, Christiansen and Barker, 1965), the density ratio (S) (Isaacs and Thodos, 1967, Chow and Adams, 2011), or the value of $\sqrt{S / E}$ for each sample (Chow and Adams, 2011). We favored the latter two strategies in this work. In addition, the Archimedes number ( $\mathrm{Ar}_{e q}$, in Eq. 5) is often used to determine particle settling owing to gravitational forces and is defined as the ratio of buoyancy and inertial forces to viscous forces acting on the object. Notice that the equivalent diameter $\left(D_{e q}\right)$ is that of a sphere of the same volume as the cylinder, and the dynamic viscosity $(\mu)$ of water is $0.001 \mathrm{~Pa} \cdot \mathrm{s}$.

$$
C_{d}=\frac{\pi}{2} \frac{\left(\rho-\rho_{w}\right) g D}{\rho_{w} u_{s}^{2}}
$$




$$
A r_{e q}=\frac{\rho_{w} D_{e q}^{3}\left(\rho-\rho_{w}\right) g}{\mu^{2}}
$$

Table 2 presents the list of cases that were simulated in this study. Cases A through E corresponded to the autonomous sensor dimensions over a prescribed range of $\sqrt{S / E}$ values; thus, the parameter $S$ did not derive from actual laboratory materials. The closest material densities would be polycarbonate (A), polyvinyl (B), teflon (C), and aluminum (D and E). Cases F through $\mathrm{J}$ corresponded to the laboratory runs labeled as $67,184,188,120$, and 20 in Isaacs and Thodos (1967), all with aspect ratio $E=2.0$. Cases $\mathrm{K}$ and $\mathrm{L}$ corresponded to nylon and aluminum with $\mathrm{E}$ $=15.6$ and 6.1, respectively, for which time-lapsed frames have been reported (Chow and Adams, 2011) and were used for qualitative comparisons of the present numerical results to laboratory findings. Case M corresponded to polyurethane at $\mathrm{E}=8.0$ (Chow and Adams, 2011). Lastly, case $\mathrm{N}$ represented the same fixed cylinder discussed in Section 3.1 but moving as a free-falling finite cylinder.

We defined the computational domain as a cylindrical tank of diameter equal to $0.56 \mathrm{~m}$ and length equal to $3 \mathrm{~m}$, which corresponded to the experimental setup in Isaacs and Thodos (1967). The cylindrical domain was discretized with $10.65 \mathrm{M}$ hexahedral cells, and the mesh was refined at the walls and floor because they corresponded to solid boundaries with a no-slip velocity condition. We ran a mesh-independence test with a coarser grid of $5.96 \mathrm{M}$ cells to simulate case A, and determined relative changes with respect to the fine mesh of $0.3 \%, 12.5 \%$, and $-12.2 \%$ in terms of the terminal velocity $\left(u_{s}\right)$, the oscillation period $\left(T_{o s c}\right)$, and the maximum inclination angle $\left(\alpha_{\max }\right)$, respectively. Thus, we maintained the fine-mesh density through all of the simulated cases in Table 2. The simulations of cylinders in free-fall mainly started from the resolution and features of the stationary cylinder cases; therefore, the mesh refinement was mostly focused on the large background mesh that represented the tank, which had a uniform cell distribution. Because we started from mesh resolutions and features known to provide good results for the fixed cylinder cases, demonstrated in section 3.1, we elected to save the computational expense of conducting a further refinement of the mesh beyond $10.65 \mathrm{M}$ cells. The top boundary was set as a symmetry plane. The core cell dimension was $8 \mathrm{~mm}$, although a refinement of $\Delta x=4 \mathrm{~mm}$ was applied over a concentric cylinder of $0.32 \mathrm{~m}$ diameter where large-velocity gradients were expected to develop due to the presence of the falling cylinder.

The overset region containing the cylinder was defined by a box of dimensions $26.4 \mathrm{~cm} \times 14.4$ $\mathrm{cm} \times 12.4 \mathrm{~cm}(\mathrm{H} \times \mathrm{W} \times \mathrm{D})$. The cylinder was horizontally positioned at $0.25 \mathrm{H}$ from the bottom of the overset region, thereby allowing for a considerable wake refinement of the mesh on the trailing side of the cylinder. The terminal velocity can be roughly estimated prior to simulating each settling event, thus giving information to ensure that the average cell size off the cylinder surface was maintained at $\mathrm{y}^{+}<1.0$. This requirement turned out to influence the accuracy of the numerical solution considerably. A gradual coarsening of the mesh was implemented to achieve a mesh topology similar to the cell distribution in the fixed cylinder test cases (Fig. 3). The time solver was discretized with a second-order scheme, and advanced at intervals of $\Delta t^{*}=$ $\Delta t \cdot u_{s} / \Delta x \approx 0.25$. Notice that in this dimensionless time step, the length scale was not defined by the cylinder diameter but instead by the background mesh refinement. This time step ensured that the solution at the overlapping cells was smoothly interpolated during four displacements before moving to the next cell layer. A hybrid second-order upwind/central differencing scheme for the convection term was selected in the segregated flow solver. The body mass and moment of inertia around the center of mass were determined from the body dimensions. The cylinders 
Table 2: Modeling data and derived quantities for falling cylinders in a tank.

\begin{tabular}{ccccccc|ccccc}
\hline \multicolumn{7}{c|}{ CFD Settings } & \multicolumn{5}{c}{ CFD Results } \\
\hline \multicolumn{1}{c}{ CaseD, cm } & $\mathrm{D}_{e q}$ & $\mathrm{~S}$ & $\mathrm{E}$ & $\sqrt{\frac{S}{E}}$ & $\mathrm{Ar}_{e q}$ & $\mathrm{Re}$ & $\mathrm{Re}_{e q}$ & $\mathrm{C}_{d}$ & $T_{\text {osc }}, \mathrm{s}$ & $\alpha_{\text {max }}{ }^{\circ}$ \\
\hline $\mathrm{A}$ & 2.40 & 4.30 & 1.245 & 3.83 & 0.570 & $1.91 \mathrm{E} 8$ & 7,454 & 13,355 & 0.941 & 1.73 & 39.5 \\
$\mathrm{~B}$ & 2.40 & 4.30 & 1.570 & 3.83 & 0.640 & $4.45 \mathrm{E} 8$ & 12,115 & 21,705 & 0.827 & 1.27 & 37.6 \\
$\mathrm{C}$ & 2.40 & 4.30 & 1.987 & 3.83 & 0.720 & $7.70 \mathrm{E} 8$ & 15,139 & 27,122 & 0.918 & 1.09 & 39.3 \\
$\mathrm{D}$ & 2.40 & 4.30 & 2.453 & 3.83 & 0.800 & $1.13 \mathrm{E} 9$ & 19,752 & 35,386 & 0.794 & 1.00 & 50.9 \\
$\mathrm{E}$ & 2.40 & 4.30 & 3.460 & 3.83 & 0.950 & $1.92 \mathrm{E} 9$ & 28,142 & 45,465 & 0.662 & 0.91 & 50.1 \\
$\mathrm{~F}$ & 2.53 & 3.65 & 1.047 & 2.01 & 0.722 & $2.24 \mathrm{E} 7$ & 3,768 & 5,444 & 0.822 & 2.69 & 52.5 \\
$\mathrm{G}$ & 2.53 & 3.65 & 1.183 & 2.01 & 0.767 & $8.76 \mathrm{E} 7$ & 7,013 & 10,132 & 0.927 & 1.45 & 56.3 \\
$\mathrm{H}$ & 1.27 & 1.83 & 1.174 & 2.00 & 0.766 & $1.05 \mathrm{E} 7$ & 2,662 & 3,839 & 0.773 & 1.05 & 53.6 \\
$\mathrm{I}$ & 1.27 & 1.83 & 2.146 & 2.00 & 1.036 & $6.91 \mathrm{E} 7$ & 6,920 & 9,981 & 0.756 & 0.55 & 58.0 \\
$\mathrm{~J}$ & 1.27 & 1.83 & 2.802 & 2.00 & 1.184 & $1.09 \mathrm{E} 8$ & 8,442 & 12,175 & 0.798 & 0.50 & 59.9 \\
$\mathrm{~K}$ & 0.96 & 2.75 & 1.140 & 15.60 & 0.270 & $2.84 \mathrm{E} 7$ & 1,500 & 4,292 & 0.848 & 2.79 & 18.5 \\
$\mathrm{~L}$ & 1.28 & 2.68 & 2.710 & 6.10 & 0.667 & $3.22 \mathrm{E} 8$ & 7,602 & 15,900 & 0.956 & 0.89 & 34.6 \\
$\mathrm{M}$ & 1.94 & 4.44 & 1.130 & 8.00 & 0.376 & $1.12 \mathrm{E} 8$ & 4,004 & 9,167 & 0.912 & 2.93 & 24.5 \\
$\mathrm{~N}$ & 2.40 & 4.02 & 1.067 & 3.14 & 0.583 & $4.31 \mathrm{E} 7$ & 4,138 & 6,937 & 0.839 & 2.76 & 46.6 \\
\hline
\end{tabular}

were released with an initial horizontal position (main axis was perpendicular to gravity and the primary motion direction).

The terminal velocity $\left(u_{s}\right)$ was determined by averaging the descending velocity as the cylinder passed at two stations in the tank. The lower station was located $0.6 \mathrm{~m}$ above the tank floor, and the upper station was set at a distance that allowed for a number of oscillations to determine secondary motion parameters. The time series of descending velocity exhibited oscillatory behavior, and the standard deviations increased with Reynolds number but remained within a range of $4-15 \%$ of $u_{s}$.

The left plot in figure 6 presents the modeling and experimental drag coefficients using the density ratio $(\mathrm{S})$ as the independent variable. Only a weak dependence can be qualitatively established between the two variables, and the $C_{d}$ moderately decreased with density ratio in both data sets. The experimental drag coefficients could qualitatively appear to exhibit a larger spread than the CFD results do, but this apparent trend arose merely due to the discrete density ratios used in the laboratory as opposed to the more gradually increasing $\mathrm{S}$ values in the simulations. The variability in $C_{d}$ emerged from both the inherent flow instability surrounding the settling cylinder, or from the need for another controlling parameter beyond the density ratio (S). The right plot in fig. 6 demonstrated the strong correlation between the Archimedes and Reynolds numbers, with a power coefficient of 0.484 that was slightly different from the value of 0.500 in the equivalent empirical correlation for spheres at large Reynolds numbers (Clift et al., 2005). Notice that the Reynolds numbers are based on the equivalent diameter $\left(\mathrm{D}_{e q}\right)$ to make the two plotted variables consistent.

The numerical results were also plotted in terms of $\sqrt{S / E}$ (in Fig. 7). The trend exhibits a decreasing behavior of $C_{d}$ with greater values of $\sqrt{S / E}$, although a conclusive correlation cannot be established given the sample size and the relatively limited range in the horizontal axis from the numerical results $(0.27-1.18)$. The experimental study (Chow and Adams, 2011) fit the data to an empirical model involving Bessel functions and dictating a tendency of $C_{d}$ to monotonically decrease. We judged that in general the present modeling results agreed satisfactorily with both laboratory data sets, and that the overset meshing technique combined with 6-DOF motion 

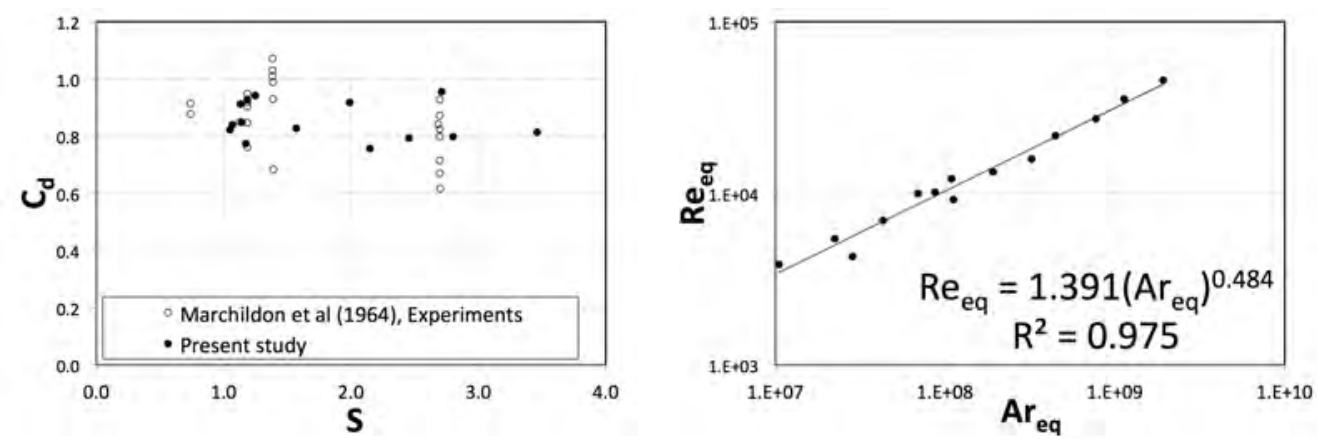

Figure 6: Drag coefficient $\left(C_{d}\right)$ plot versus the density ratio (S) (left) and log-scale plot of $\mathrm{Re}_{e q}$ versus Archimedes number $\left(A r_{e q}\right)$ (right)

solving is an effective method for resolving the primary motion metrics in settling cylinders.

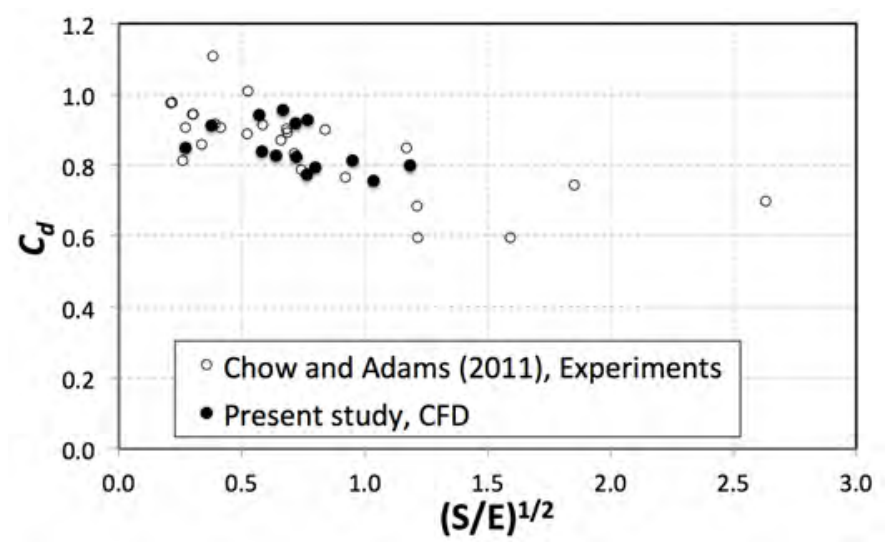

Figure 7: Drag coefficient $\left(C_{d}\right)$ plot versus $\sqrt{S / E}$.

\subsection{Freely settling cylindrical particles: secondary motion}

Secondary motion is related to the oscillatory behavior that the cylinders exhibit while settling. This type of angular movement is the manifestation of the effects of periodic vortex shedding emerging from the cylinder surface. This periodicity gives rise in turn to oscillatory fluid forcing running laterally along the cylinder axis, resembling the three-dimensionality found in the fixed cylinder tests (Spalart, 2009). Observations of the settling cylinders in the present simulations showed an absence of secondary motion during the initial time lasting from release to reaching $u_{s}$ (approximately $t^{*}<80$ in Fig. 8). During this period, the lateral forcing is developing but is not unbalanced enough to produce angular displacement, similar to the manner in which vortex shedding in a fixed cylinder develops after an initial warm-up period when either the experiments or simulations start with the fluid at rest. However, in the settling cylinder, no motion component is suppressed, and the cylinder reacts to the forcing by exhibiting angular and lateral displacements. In the numerical simulations, the onset of the secondary motion lowers and induces a periodic behavior of the $u_{s}$. 


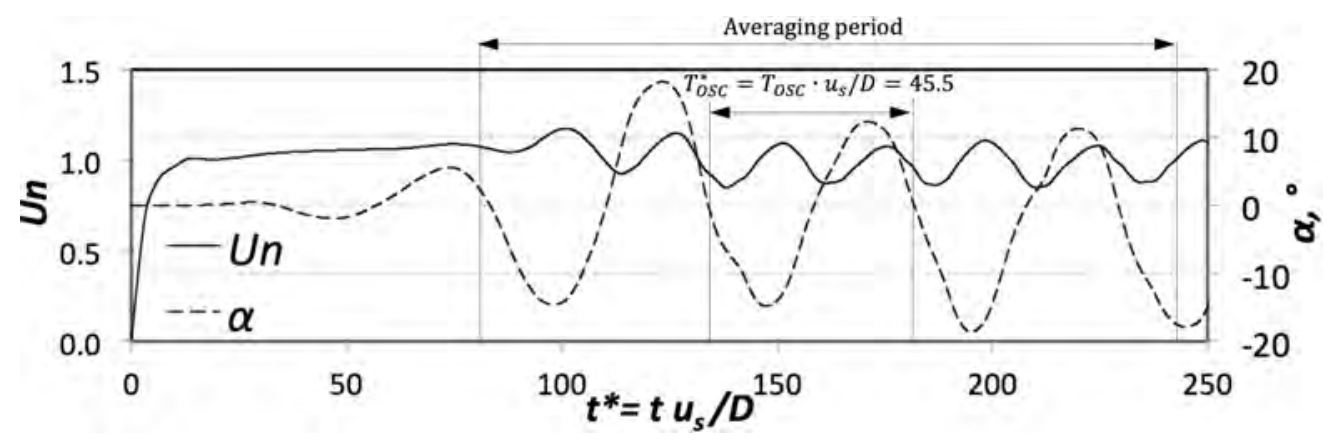

Figure 8: Time series of the normalized downwards velocity (Un) and lateral displacement of the center of mass ( $\left.Z^{*}\right)$ for case $\mathrm{K}$ in Table 2

As the cylinder fell and acquired a periodic angular movement, the solution monitored the time series of the orientation of the main axis with respect to a horizontal line $(\alpha)$. One half of $T_{o s c}$ (Fig. 8) represented the lapse between two consecutive occurrences when the body was in a horizontal orientation. The empirical equations (6) and (7) were proposed and tested in laboratory studies and showed considerable agreement with experimental measurements of oscillation period (Marchildon et al., 1964, Chow and Adams, 2011). The numerical values of $T_{o s c}$ exhibited a small variation at each cycle as the cylinder traveled between the two reference stations (approximately $80<t *<240$ in fig. 8). For that reason, we computed the power spectrum of the time series of $\alpha$ by means of Fast Fourier Transform (FFT) functions, and $T_{o s c}$ was thus the inverse of the frequency at maximum power. We compared both the empirical values based on Eq. 6 and the CFD outcomes in Fig. 9. We observed a considerable correlation between the empirical and CFD results, although some deviations from the bisecting line can be observed in individual particles at large $T_{o s c}$ values. From the numerical sample, these large-oscillation runs corresponded to either large aspect ratio values (E) or low-density ratios (S), both instances resulting in low terminal velocities and low Reynolds numbers. Qualitative inspections of experimental results also indicate a larger spread at large $T_{\text {osc }}$ (Marchildon et al., 1964, Chow and Adams, 2011), thereby suggesting that at low Reynolds number, Eq. 6 might not be accounting for other effects in the angular force balance from which it derives:

$$
T_{o s c}=7.91 \cdot \sqrt{\frac{S L}{g^{\prime}}}
$$

where the reduced gravity of the particle is

$$
g^{\prime}=\frac{\left(\rho-\rho_{w}\right) g}{\rho_{w}}
$$

Values of $\alpha_{\max }$ are plotted in Fig. 10. Based on this motion parameter, three modes of secondary motion have been identified: (i) oscillation only $(\sqrt{S / E}<1.2)$, (ii) oscillation/tumbling $(1.2<\sqrt{S / E}<2.0)$, and (iii) tumbling only $(\sqrt{S / E}>2.0)$. The cases with low aspect ratio $(L=2.0)$ resulted in the largest values of $\alpha_{\max }$, a pattern previously discussed in a laboratory work (Isaacs and Thodos, 1967). Although all of the simulation runs remained confined within the "oscillation only" mode $(\sqrt{S / E}<1.2)$, a few instances exhibited tumbling in the last portion of travel. This fact suggested that tumbling is likely a manifestation of further flow instabilities 


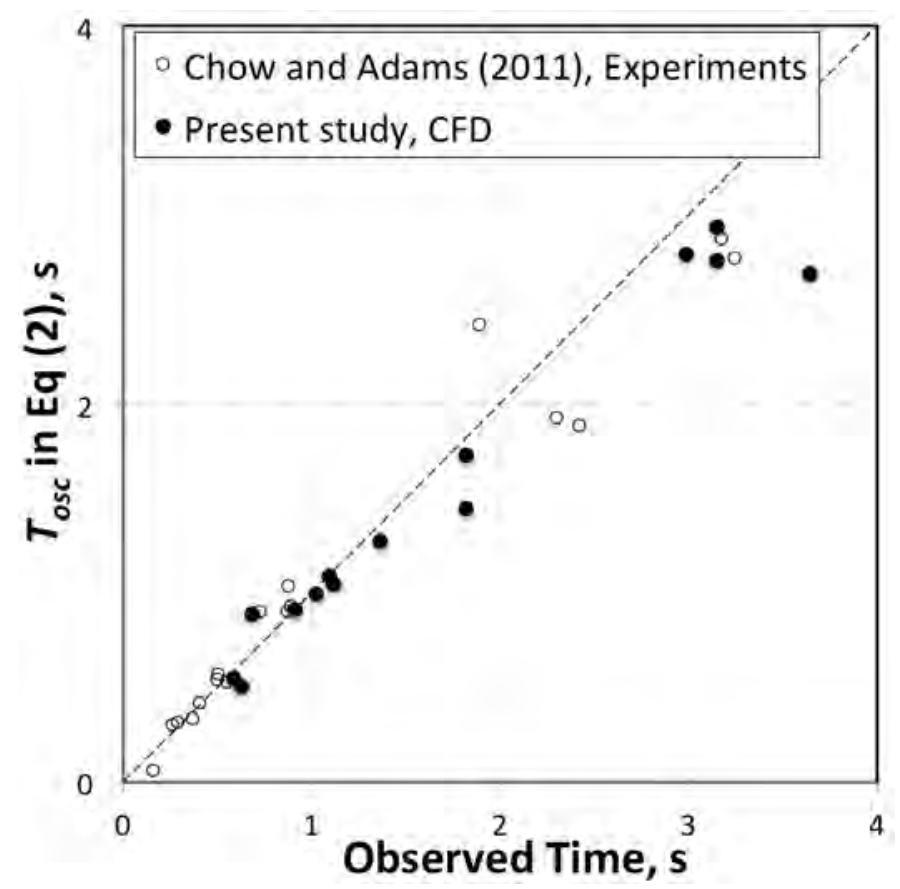

Figure 9: Oscillatory frequency of cylinders: experiments from (Chow and Adams, 2011) and CFD results in the present study, compared to $\mathrm{T}_{\text {osc }}$ calculated from Eq. 6.

developing around the particle during the "oscillation only" mode, and may arise fully if the cylinder were allowed to travel continuously without reaching the tank floor (i.e., in an infinitely long tank). However, this hypothesis should be put to the test with further simulations in enlarged tank dimensions.

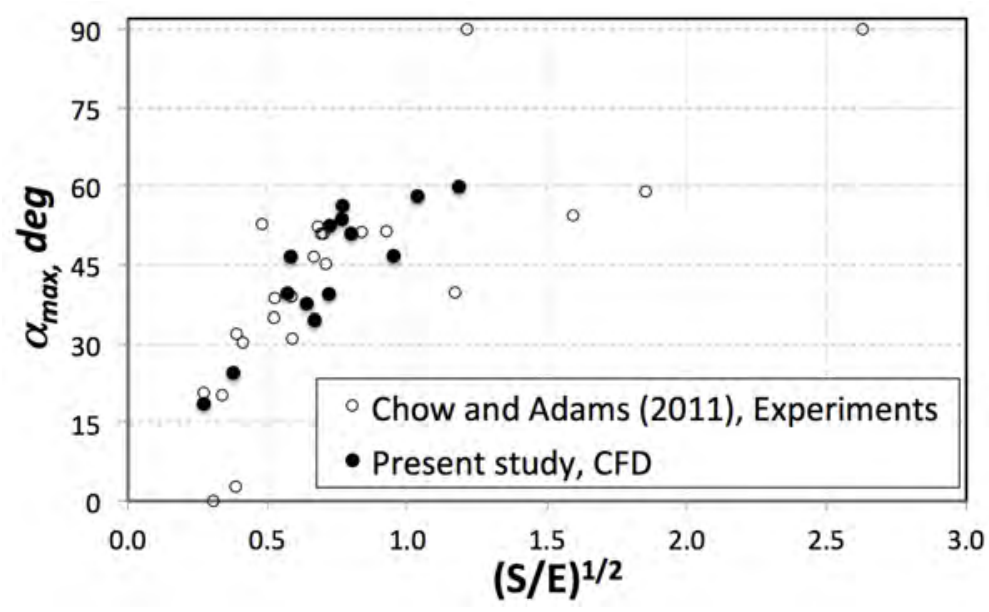

Figure 10: Plot of the maximum particle deflection from horizontal $\alpha_{\max }$ versus $\sqrt{S / E}$. 
To establish qualitatively comparisons of the simulated movement of the cylinder against laboratory images (Chow and Adams, 2011), Fig. 11 depicts the superposition of frames at various instants. At travel times $t^{*}=t \cdot u_{s} / D=100$ and 120 , the cylinder has fully developed the secondary oscillations. Figure 11 demonstrates that the CFD approach presented in this study can adequately represent the motion of settling large-sized cylinders in quiescent fluids. The instantaneous frames from both techniques are arranged side-by-side (Fig. 11, left) and overlapped (Fig. 11, right). Notice that both the angular displacement and lateral displacement (in z-direction) are correctly reproduced by the modeling approach.
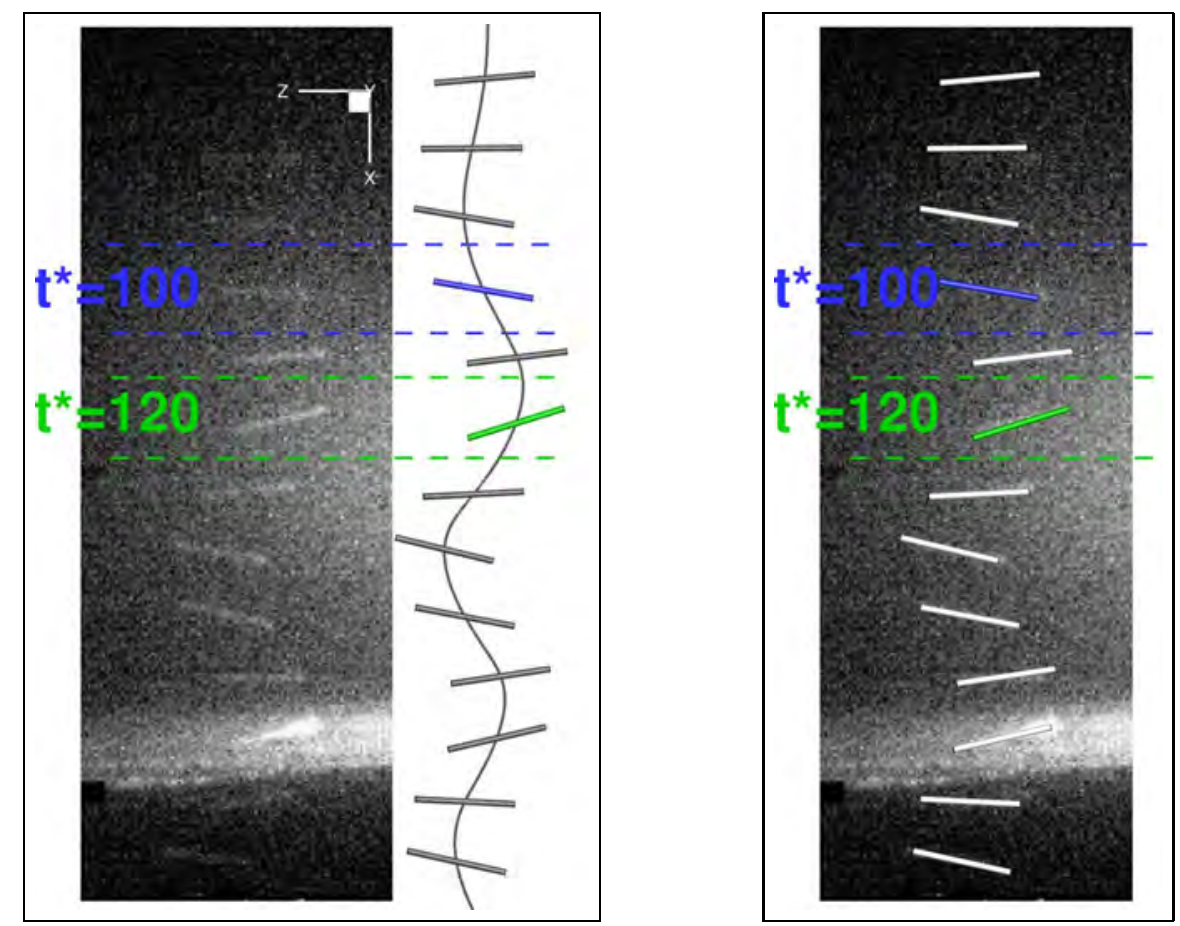

Figure 11: Time-lapsed frames at 0.62-s intervals for case $\mathrm{K}$ in Table 2. The background image was taken from Chow and Adams (2011). The CFD results show the trajectory of the cylinder centroid (left), and the laboratory image and CFD locations are overlapped (right). The flow conditions at two instants are further examined, $t^{*}=t \cdot u_{s} / D=100$ and 120

Features of the simulated flow field surrounding the cylinder at three instants in fig. 12. At an earlier stage at $t^{*}=50$, the falling cylinder shows fully developed terminal velocity but developing secondary motion; the actual locations of the cylinder at $t^{*}=100$ and 120 were shown in Fig. 11. Figure 12 combines the contours of the normalized z-vorticity and contours of the pressure coefficient on the cylinder surface. The z-vorticity contour lines were normalized by defining $\left|\omega_{z} D / U_{0}\right|_{\min }=1.0$ and $\left.\left|\Delta \omega_{z} D / U_{0}\right|=0.5\right)$ at three planes normal to the z-direction located at $20 \%, 50 \%$, and $80 \%$ of the cylinder length. The three instants present the same type of shear layer separation at the wake as shown in the fixed cylinder test case (Fig. 4), except that the symmetry was lost because the vorticity is instantaneous and the cylinder presents an angle of inclination $(\alpha)$. Although dynamic, the shear layer maintains a relatively similar extent both along the cylinder body, and across the three instants. This consistent pattern of the shear layer 
imprints on the cylinder an oscillatory behavior that is sustained for a considerable period. The pressure coefficients also exhibit maximum values on the leading half and "lifted" side of the cylinder (on the most negative x-coordinate values). Notice that high values of $C_{p}$ are present in the z-positive half of the cylinder at $t^{*}=100$ but lie in the z-negative half at $t^{*}=120$, whereas $C_{p}$ remains fairly uniform at $t^{*}=50$ when no considerable $\alpha$ value is observed. Figure 13 presents contours of normalized velocity magnitude showing that the flow disturbance is laterally confined within the domain corresponding to the overset region, and that a long trailing wake stretches behind the cylinder. The latter feature is equivalent to the vortex shedding that continuously propagates in the wake of flows past fixed cylinders. In spite of the transient behavior, the apparent symmetry at $t^{*}=50$ (with no oscillations yet present) is remarkably different from the other two frames where the secondary motion has set in.

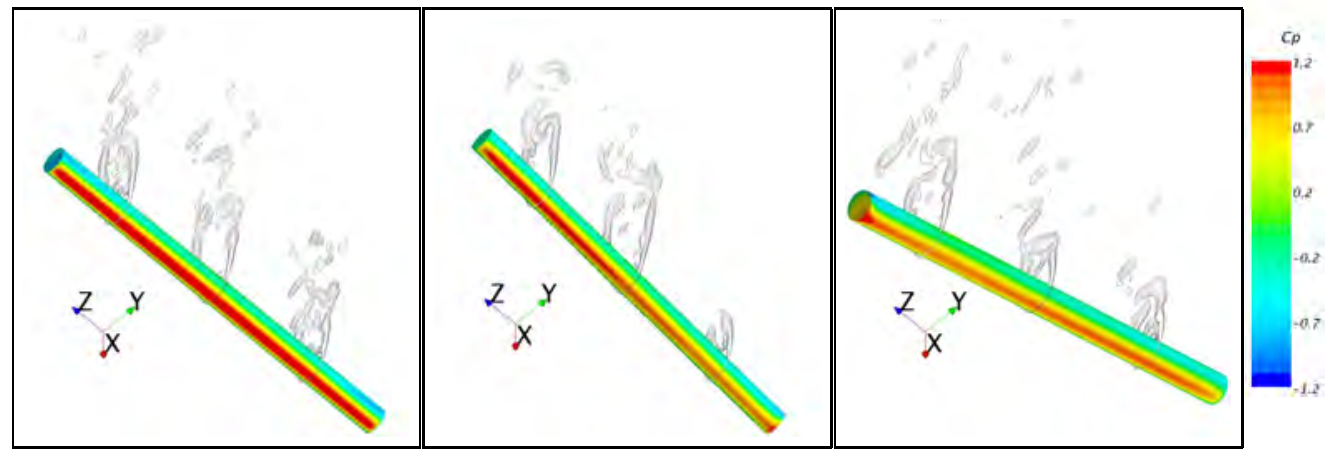

Figure 12: Flow fields in three instants $\left(t^{*}=t \cdot u_{s} / D\right)$ : 50 (left), 100 (center), and 120 (right). Contours of surface pressure coefficients are defined with the surface-averaged pressure $\left(P_{\text {ave }}\right)$ and the terminal velocity $\left(u_{s}\right)$ in Table 2 for case $\mathrm{K}, C_{p}=\left(P-P_{\text {ave }}\right) /\left({ }^{1} /{ }_{2} \rho_{w} u_{s}\right)$.

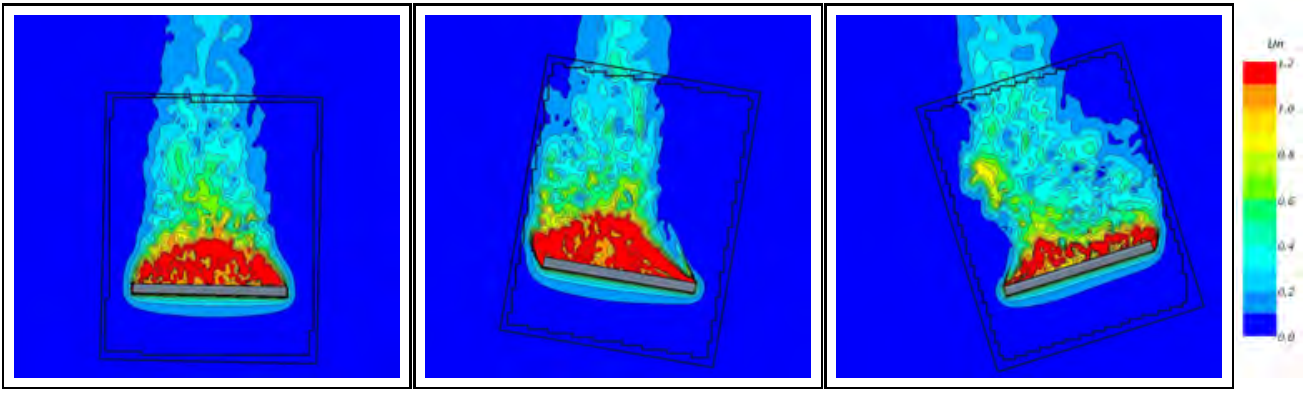

Figure 13: Normalized $\mathrm{x}$-velocity contours $\left(U n=u / u_{s}\right)$ at three instants $\left(t^{*}\right): 50$ (left), 100 (center) and 120 (right).

In view of this agreement, we expect that the present method can be expanded to other conditions not pursued in this study, but that are relevant in other practical situations. For instance, this approach is amenable to irregular shapes that are more prevalent in environmental settings rather than the regular geometry examined here. In another instance, other forms of secondary motion 
at higher Reynolds numbers or particles subjected to active flows can be analyzed by gradually increasing the level of complexity. Studies of reactive particles can benefit from the present approach because effective reaction rates depend on the flow conditions surrounding the particles. Thus, the experimental design can be conducted by first describing the fluid environment around the particle in motion, to later run the chemical reactions on accurate flow fields. However, these benefits must be evaluated with respect to the cost of having access to the flow fields at any prescribed time. Each of the present simulations took approximately 72 hours of clock time running on 6 nodes of $64 \mathrm{~GB} 1,600 \mathrm{MHz}$ memory each (32 cores per node, AMD Interlagos $2.1 \mathrm{GHz}$ ). Notice that while the time step was optimized for computational expense, the mesh size and parallel cluster selection were not (the largest mesh size from the mesh-independence test was used, and other parallel clusters at our institution have shown better performance). Nevertheless, we recognized that the detailed characterization of flows around moving objects with CFD is a time-consuming task, the use of which must be carefully assessed in each intended application. One can expect the computational effort to increase when dealing with Reynolds numbers higher than those investigated in this work.

\section{Conclusions}

This study simulated the motion of settling cylinders in quiescent water by means of computing the transient flow conditions surrounding the cylinder. The method combined (i) an overset grid, (ii) a 6-DOF motion solver, and (iii) an eddy-resolving flow simulation approach into a CFD software tool. The results were reported in terms of the drag coefficients at terminal velocity, body oscillatory frequency, and maximum angle of inclination. The simulated results showed an acceptable agreement with published laboratory data conducted using cylindrical particles of relatively large size as was done in this study. Qualitatively, the simulated body motion also reproduced the features of a test case for which laboratory imaging was available.

The most critical aspect of this work is the computational expense incurred, which can currently be considered large or not readily available for all technical groups interested in this type of engineering application. Nevertheless, the present study demonstrated benefits, namely, the ability to yield full descriptions of the flow fields arising from the fluid-body interactions at each solution time, as well as to modify various parameters controlling the phenomenon. The present method can aid in developing technology for engineering applications, such as: (i) the design of sensor devices that monitor the flow environment but whose recordings in turn are influenced by the sensor motion, and (ii) the development of reactive particles the efficiency of which will be affected by the flow conditions surrounding the cylinders. Although computationally expensive, the present approach can be used during the design stage before instrumenting devices and equipment to be deployed in environmental flows.

\section{Acknowledgments}

This research was supported by the U.S. Department of Energy, Energy Efficiency and Renewable Energy, Wind and Water Power Program.

Computations described here were performed using the facilities of the Pacific Northwest National Laboratory (PNNL) institutional computing center (PIC).

Pacific Northwest National Laboratory (PNNL) is operated for the U.S. Department of Energy by Battelle under Contract No. DE-AC06-76RLO 1830. 


\section{References}

\section{References}

Carlson, T. J., Duncan, J. P., Gilbride, T., 2003. The sensor fish: measuring fish passage in severe hydraulic conditions. Hydro Review 22 (6), 62-69.

CD-adapco, 2014. User Guide, STAR-CCM+ Version 9.06. CD-adapco, http://www.cd-adapco.com.

Chow, A., Adams, E., 2011. Prediction of drag coefficient and secondary motion of free-falling rigid cylindrical particles with and without curvature at moderate Reynolds number. Journal of Hydraulic Engineering 137 (11), 1406-1414.

Christiansen, E. B., Barker, D. H., 1965. The effect of shape and density on the free settling of particles at high reynolds numbers. AIChE Journal 11 (1), 145-151.

Chu, P. C., Gilles, A., Fan, C., 2005. Experiment of falling cylinder through the water column. Experimental Thermal and Fluid Science 29 (5), $555-568$.

Clift, R., Grace, J. R., Weber, M. E., 2005. Bubbles, drops, and particles. Courier Corporation, New York, NY.

Deng, Z., Carlson, T. J., Duncan, J. P., Richmond, M. C., 2007. Six-degree-of-freedom sensor fish design and instrumentation. Sensors 7 (12), 3399-3415.

Dietrich, W. E., 1982. Settling velocity of natural particles. Water resources research 18 (6), 1615-1626.

Dong, S., Karniadakis, G. E., Ekmekci, A., Rockwell, D., 12 2006. A combined direct numerical simulation-particle image velocimetry study of the turbulent near wake. Journal of Fluid Mechanics 569, 185-207.

Dougherty, F. C., 1985. Development of a chimera grid scheme with applications to unsteady problems. Ph.D. thesis, Stanford University, Palo Alto, CA.

Ferziger, J. H., Perić, M., 2002. Computational methods for fluid dynamics. Vol. 3. Springer Berlin.

Hashino, T., Chiruta, M., Polzin, D., Kubicek, A., Wang, P. K., 2014. Numerical simulation of the flow fields around falling ice crystals with inclined orientation and the hydrodynamic torque. Atmospheric Research 150 (0), 79 - 96.

Hinatsu, M., Ferziger, J. H., 1991. Numerical computation of unsteady incompressible flow in complex geometry using a composite multigrid technique. International Journal for Numerical Methods in Fluids 13 (8), 971-997.

Isaacs, J. L., Thodos, G., 1967. The free-settling of solid cylindrical particles in the turbulent regime. The Canadian Journal of Chemical Engineering 45 (3), 150-155.

Jayaweera, K. O. L. F., Mason, B. J., 8 1965. The behaviour of freely falling cylinders and cones in a viscous fluid. Journal of Fluid Mechanics 22, 709-720.

Kravchenko, A. G., Moin, P., 2000. Numerical studies of flow over a circular cylinder at Re=3900. Physics of Fluids (1994-present) 12 (2), 403-417.

Luo, D., Yan, C., Liu, H., Zhao, R., 2014. Comparative assessment of PANS and DES for simulation of flow past a circular cylinder. Journal of Wind Engineering and Industrial Aerodynamics 134 (0), 65 - 77.

Mandø, M., Rosendahl, L., 2010. On the motion of non-spherical particles at high reynolds number. Powder Technology $202(1-3), 1-13$.

Marchildon, E. K., Clamen, A., Gauvin, W. H., 1964. Drag and oscillatory motion of freely falling cylindrical particles. The Canadian Journal of Chemical Engineering 42 (4), 178-182.

McNown, J. S., Malaika, J., 1950. Effects of particle shape on settling velocity at low reynolds numbers. Eos, Transactions American Geophysical Union 31 (1), 74-82.

Menter, F., Kuntz, M., 2004. Adaptation of eddy-viscosity turbulence models to unsteady separated flow behind vehicles. In: McCallen, R., Browand, F., Ross, J. (Eds.), The Aerodynamics of Heavy Vehicles: Trucks, Buses, and Trains. Vol. 19 of Lecture Notes in Applied and Computational Mechanics. Springer Berlin Heidelberg, pp. 339-352.

Menter, F. R., 1994. Two-equation eddy-viscosity turbulence models for engineering applications. AIAA journal 32 (8), $1598-1605$.

Norberg, C., 1987. Effects of Reynolds number and a low-intensity freestream turbulence on the flow around a circular cylinder. Chalmers University, Goteborg, Sweden, Technological Publications 87 (2).

Ren, B., Zhong, W., Jin, B., Lu, Y., Chen, X., Xiao, R., 2011. Study on the drag of a cylinder-shaped particle in steady upward gas flow. Industrial \& Engineering Chemistry Research 50 (12), 7593-7600.

Richmond, M., Serkowski, J., Rakowski, C., Strickler, B., Weisbeck, M., Dotson, C., 2014. Computational Tools to Assess Turbine Biological Performance. Hydro Review 33 (6).

Richmond, M. C., Deng, Z., McKinstry, C. A., Mueller, R. P., Carlson, T. J., Dauble, D. D., 2009. Response relationships between juvenile salmon and an autonomous sensor in turbulent flow. Fisheries Research 97 (1), 134-139.

Shim, Y. M., Sharma, R. N., Richards, P., 2009. Numerical study of the flow over a circular cylinder in the near wake at Reynolds number 3900. In: 39th AIAA Fluid Dynamics Conference. pp. AIAA 2009-4160.

Spalart, P. R., 2009. Detached-Eddy Simulation. Annual Review of Fluid Mechanics 41, 181-202.

Stringham, G., Simons, D. B., Guy, H. P., 1969. The behavior of large particles falling in quiescent liquids. US Government Printing Office, Washington, DC. 
Travin, A., Shur, M., Strelets, M., Spalart, P., 2002. Physical and numerical upgrades in the detached-eddy simulation of complex turbulent flows. In: Advances in LES of complex flows. Springer, pp. 239-254.

Young, M., Ooi, A., 2007. Comparative assessment of les and urans for flow over a cylinder at a Reynolds number of 3900. In: 16th Australasian Fluid Mechanics Conference (AFMC). School of Engineering, The University of Queensland, pp. 1063-1070. 